\title{
Is mental practice effective for treating upper extremity deficits in individuals with hemiparesis after stroke? A Cochrane Review summary with commentary
}

\author{
Francesca Gimigliano \\ Department of Mental and Physical Health and Preventive Medicine, University of Campania "Luigi Vanvitelli", \\ Via de Crecchio, 4, Naples, Italy \\ Tel.: +39 349 3185769; E-mail: francescagimigliano@gmail.com.
}

\begin{abstract}
.
BACKGROUND: Mental practice, which is proposed for the rehabilitation of people post-stroke, is a training method based on the repetition of the internal representation of a movement or a task with the aim of improving the performance.

OBJECTIVE: The aim of this commentary is to discuss Cochrane evidence on the efficacy of mental practice in improving upper extremity functioning in people with hemiparesis after stroke.

METHODS: To summarize and discuss from a rehabilitation perspective the published Cochrane Review "Mental practice for treating upper extremity deficits in individuals with hemiparesis after stroke" by Barclay et al.

RESULTS: This Cochrane Review included 25 studies involving 676 people with hemiparesis after stroke. The authors analysed the following two comparisons: mental practice versus conventional therapy and mental practice in addition to other treatment versus other treatment ( \pm placebo).

CONCLUSIONS: Mental practice in addition to other treatment, compared with other treatment, probably improves upper extremity activity and function in people with hemiparesis after stroke.
\end{abstract}

Keywords: Mental practice, hemiparesis, stroke, rehabilitation, activity, functioning

The aim of this commentary is to discuss the Cochrane Systematic Review "Mental practice for treating upper extremity deficits in individuals with hemiparesis after stroke" (Barclay et al., 2020) by Barclay, Stevenson, Poluha, Semenko, and Schubert, ${ }^{1}$ published under the direct supervision

\footnotetext{
${ }^{1}$ This summary is based on a Cochrane Review previously published in the Cochrane Database of Systematic Reviews 2020, Issue 5, Art. No.: CD005950, DOI: 10.1002/14651858.CD005950. pub5 (see www.cochranelibrary.com for information). Cochrane Reviews are regularly updated as new evidence emerges and in response to feedback, and Cochrane Database of Systematic
}

of Cochrane Stroke Group, from a rehabilitation perspective. This Cochrane Corner is produced in agreement with NeuroRehabilitation by Cochrane Rehabilitation.

Reviews should be consulted for the most recent version of the review.

The views expressed in the summary with commentary are those of the Cochrane Corner author and do not represent the Cochrane Library or Wiley. 


\section{Background}

A stroke is the sudden occurrence of permanent damage to an area of the brain caused by a blocked blood vessel or bleeding within the brain. It is the second leading cause of death of people worldwide, and it is the top cause of the need for rehabilitation among patients with neurological disorders around the world (Cieza et al., 2020). Rehabilitation as the "medicine of functioning" is described by the International Classification of Functioning, Disability and Health (ICF) and it has been recently considered as the key health strategy of the 21st century addressing all chronic disabling conditions (Gimigliano et al., 2017).

Mental practice is a training method that involves the repetition of the internal representation of a movement or a task with the aim of improving the performance. The recovery of upper extremity functioning is critical in the rehabilitation of patients post stroke, and information about the efficacy and safety of mental practice would be of major importance for rehabilitation professionals to plan an adequate individualized rehabilitative programme.

\section{Mental practice for treating upper extremity deficits in individuals with hemiparesis after stroke}

(Barclay RE, Stevenson TJ, Poluha W, Semenko B, Schubert J, 2020)

\section{Objective}

The aim of this Cochrane Systematic Review was to determine whether mental practice improves outcomes relating to upper extremity functioning in people with hemiparesis after stroke.

\section{What was studied and methods}

The population addressed in this review were adult stroke survivors with limitations in upper extremity activity. The intervention studied was mental practice alone or in addition to other treatment. The intervention was compared to no intervention, conventional therapy, placebo mental practice, placebo mental practice with conventional therapy, and other therapeutic interventions. The primary outcomes of interest were activity and activity limitations of the upper extremity (measured by tools such as Box and
Block test, Action Research Arm Test, Wolf Motor Function Test, and Motor Activity Log), and hand function (measured by tools such as Jebsen Test of Hand Function). Secondary outcomes of interest were changes in body structure or function of the upper extremity (e.g. Fugl-Meyer Test of Sensorimotor Ability, and Impairment Inventory of the Chedoke-McMaster Stroke Assessment), activities of daily living (e.g. Barthel Index, and Functional Independence Measure), health-related quality of life (e.g. Stroke Impact Scale), economic costs of the interventions, and adverse effects (including death).

Searching for randomised controlled studies was carried out in September 2019. Study selection, quality assessment, data extraction, analysis and synthesis were conducted using Cochrane methods.

\section{Results}

This Cochrane Review included 25 studies involving 676 people with hemiparesis after stroke. The review authors analysed the following two comparisons.

1. Mental practice compared to conventional therapy:

- Three trials (50 people with hemiparesis after stroke) examined the outcome of upper extremity impairment and reported no difference between groups (SMD 0.34, $95 \% \mathrm{CI}-0.33$ to $1.00 ; \mathrm{I}^{2}=21 \%$; low quality evidence).

Data on other outcomes were not available for meta-analysis.

2. Mental practice in addition to other treatment compared to other treatment ( \pm placebo):

- Fifteen trials (397 people with hemiparesis after stroke) examined the outcomes of upper extremity activity and impairment. Better results were reported for the group with added mental practice (for upper extremity activity: SMD $0.66,95 \%$ CI 0.39 to $0.94 ; \mathrm{I}^{2}=39 \%$; moderate quality evidence; for upper extremity impairment: SMD $0.59,95 \%$ CI 0.30 to $0.87 ; \mathrm{I}^{2}=$ $43 \%$; moderate quality evidence). Subgroup analyses explored the time post stroke, dosage of training, and type of comparison, and no differences were found.

- Four trials (157 people with hemiparesis after stroke) examined the activities 
of daily living and reported no difference between groups (SMD 0.08, 95\% CI -0.24 to $0.39 ; \mathrm{I}^{2}=0 \%$; low quality evidence).

- Data on other outcomes were not available for meta-analysis.

\section{Conclusions}

For people with hemiparesis after stroke, mental practice in addition to other treatment compared with other treatment, probably improve upper extremity activity and function. However, it may make no difference in the use of the paretic arm in the activities of daily living. Moreover, mental practice alone compared to conventional treatment may make no difference in upper extremity function. Data on other comparisons or outcomes are not available.

\section{Implications for practice in neurorehabilitation}

The recovery of the upper extremity functioning is always challenging in people post-stroke. Nonetheless, the addition of mental practice to conventional therapy seems effective in enhancing arm functioning in people with hemiparesis after stroke and should thus be considered when planning their individualized rehabilitative programme. Additionally, high-quality studies need to be conducted to elucidate the effects of mental practice on activities of daily living, health-related quality of life, and economic costs and to determine the optimal treatment protocol and patient profile among individuals with hemiparesis after stroke.

\section{Acknowledgments}

The author thanks Cochrane Rehabilitation and Cochrane Stroke Group for reviewing the contents of the Cochrane Corner.

\section{Conflict of interest}

The author declares no conflicts of interest.

\section{References}

Barclay, R. E., Stevenson, T. J., Poluha, W., Semenko, B., \& Schubert, J. (2020). Mental practice for treating upper extremity deficits in individuals with hemiparesis after stroke. The Cochrane Database of Systematic Reviews, 5(5), CD005950. https://doi.org/10.1002/14651858.CD005950.pub5

Cieza, A., Causey, K., Kamenov, K., Hanson, S. W., Chatterji, S., \& Vos, T. (2021). Global estimates of the need for rehabilitation based on the Global Burden of Disease study 2019: a systematic analysis for the Global Burden of Disease Study 2019. Lancet (London, England), 396(10267), 2006-2017. https://doi.org/10.1016/S0140-6736(20)32340-0

Gimigliano, F., \& Negrini, S. (2017). The World Health Organization "Rehabilitation 2030: a call for action". European Journal of Physical and Rehabilitation Medicine, 53(2), 155168. https://doi.org/10.23736/S1973-9087.17.04746-3 